\title{
Comparison of Sampler Filling Times Among Selected Arterial Blood Samplers
}

\author{
Christine N McMillen RRT, Stephen Adu-Yeboah RRT, Elisabeth Kagarise RRT, \\ Rebecca L Morton RRT, Basak Yuce RRT, and F Herbert Douce MSc RRT-NPS RPFT FAARC
}

\begin{abstract}
BACKGROUND: True arterial blood samples are essential in making clinical decisions for respiratory patients. Previous studies using only the Portex Pro-Vent arterial sampler have shown a significant difference between arterial and venous filling times. The goal of this study was to determine whether there is a statistically significant difference between sampler filling times measured at a normal mean arterial pressure among multiple arterial samplers with plungers and to determine whether there is a statistically significant difference in filling times between venous and normal mean arterial pressures for a sampler without a plunger. METHODS: We assembled an extracorporeal laboratory model to circulate a synthetic compound composed of $0.9 \%$ sodium chloride solution and Life/form artificial blood, and we used hemostats to create pressures within the circuit. We randomly selected samplers and measured the filling times of $\mathbf{4}$ arterial samplers with plungers at a normal mean arterial pressure $(93 \pm 1 \mathrm{~mm} \mathrm{Hg})$. We also measured the filling time of one arterial sampler without a plunger at a normal mean arterial pressure and at a simulated venous pressure $(9 \pm 2 \mathrm{~mm} \mathrm{Hg})$. We used the Kruskal-Wallis one-way analysis of variance to compare arterial filling times in samplers, and we used a $t$ test for independent samples to compare venous and arterial filling times in the sampler without a plunger. RESULTS: There was a statistically significant difference between sampler filling times among the $\mathbf{4}$ arterial samplers with a plunger $(P<.001)$. There was a statistically significant difference between arterial and venous pressure filling times for the sampler without a plunger $(P<.001)$. CONCLUSIONS: Although there was a statistically significant difference between arterial filling times among various samplers with plungers, the difference was $<1 \mathrm{~s}$ and was not deemed clinically important. Regardless of the sampler brand being used, respiratory therapists and other clinicians performing arterial punctures can use sampler filling time to identify a successful arterial puncture while drawing blood. Key words: arterial blood sampling; percutaneous puncture; venous blood; arterial sampler; syringes; filling time; arterial blood pressure. [Respir Care 2015;60(4):513-516. (C) 2015 Daedalus Enterprises]
\end{abstract}

\section{Introduction}

In most hospitals, clinicians obtain arterial blood samples to monitor acid/base status and adequacy of ventila-

Ms McMillen is affiliated with the Division of Respiratory Care, Cincinnati Children's Hospital Medical Center, Cincinnati, Ohio. Mr AduYeboah is affiliated with the Ross University School of Medicine, Roseau, Commonwealth of Dominica, West Indies. Ms Kagarise is affiliated with the Respiratory Care Department, Berger Hospital, Circleville, Ohio. Ms Morton and Ms Yuce are affiliated with the Respiratory Therapy Department, Wexner Medical Center, and Mr Douce is affiliated with the Respiratory Therapy Division, School of Health and Rehabilitation Sciences, College of Medicine, The Ohio State University, Columbus, Ohio. tion and oxygenation to assess patients' cardiopulmonary condition. Based on arterial blood gas results, clinical decisions are made regarding ventilator settings, oxygen therapy, respiratory treatments, and changes in clinical condi-

\footnotetext{
At the time this study was conducted, all authors were associated with the Respiratory Therapy Division, School of Health and Rehabilitation Sciences, College of Medicine, The Ohio State University, Columbus, Ohio.

Ms McMillen presented a version of this paper at the OPEN FORUM of the AARC Congress 2013, held November 16-19, 2013, in Anaheim, California.
}

The authors have disclosed no conflicts of interest. 
tion. Currently, a blood flash in the hub indicates to the medical professional that an arterial puncture is successful. However, because both venous and arterial sticks can provide an initial blood flashback in the hub, relying exclusively on this flash as being indicative of an arterial stick is insufficient.

When arterial blood is obtained via percutaneous puncture, arterial sampler filling time has been shown to be a useful indicator identifying the sample as arterial. In a laboratory study, Johnson et al ${ }^{1}$ demonstrated significant differences between arterial and venous filling times regardless of needle size. Bender et $\mathrm{al}^{2}$ studied sampler filling times in human subjects with normal mean arterial pressures and concluded that arterial puncture filling time is significantly faster than venopuncture. Cortes et $\mathrm{al}^{3}$ showed that there was no statistically significant difference in arterial filling times for subjects with abnormal blood pressures. Although these studies showed that sampler filling time is a useful indicator that the sample is arterial, all 3 studies used only the Portex Pro-Vent arterial sampler (Smiths Medical, Dublin, Ohio) in their comparisons. Other arterial sampler brands may perform differently.

If filling time is excessive, blood exposed to atmospheric air might impact blood gas results. ${ }^{4}$ Because a significant difference in filling times between arterial and venous pressures was already established in a sampler with a plunger, the aim of this study was to determine whether there is a statistically significant difference between sampler filling times measured at a normal mean arterial pressure among every currently available sampler with a plunger in the United States, including the previously used Portex ProVent, and to determine whether there is a statistically significant difference in filling times between venous and normal mean arterial pressures for the only available sampler without a plunger in the United States. We hypothesized that there would not be a significant difference between filling times among the selected samplers with plungers. We also hypothesized that there would be a statistically significant difference between arterial and venous filling times for the sampler without a plunger.

\section{Methods}

This study was conducted in the Respiratory Therapy Research Laboratory of the School of Health and Rehabilitation Sciences at The Ohio State University in Columbus, Ohio. We performed a comparative laboratory experiment to assess the filling times of 4 arterial samplers with plungers and one arterial sampler without a plunger. As a

Correspondence: Christine N McMillen RRT, E-mail: cmcmillen.64@ gmail.com.

DOI: $10.4187 /$ respcare. 03480

\section{QUICK LOOK}

\section{Current knowledge}

Arterial puncture to obtain blood for arterial blood gas analysis is a common procedure performed by respiratory therapists. Assuring that the sample is arterial as opposed to venous is critical for providing meaningful data for patient management. A flash in the hub of the needle, blood color, pulsatile flow, and syringe filling times are all used to indicate arterial blood.

\section{What this paper contributes to our knowledge}

Arterial punctures can be accomplished using any currently available vented arterial blood gas sampler, and successful arterial puncture can be confirmed based on filling times or rate $(\mathrm{s} / \mathrm{mL})$. There were statistically significant differences between arterial filling times among various devices; the differences were $<1 \mathrm{~s}$ and were not deemed clinically important.

substitute for whole blood, we used a synthetic compound composed of $0.9 \%$ sodium chloride solution and Life/form artificial blood (Nasco, Fort Atkinson, Wisconsin). We assembled a circulation model similar to that of Johnson et $\mathrm{al}^{1}$ with an extracorporeal blood pump speed control (Bio-Console 540, Medtronic Bio-Medicus, Eden Prairie, Minnesota) set to circulate the synthetic blood at a constant flow of $1.16 \mathrm{~L} / \mathrm{min}$. We used a heat exchanger (CAPIOX RX05 Baby RX, Terumo, Ann Arbor, Michigan) and a Haake heater to maintain $37^{\circ} \mathrm{C}$ within the circuit. We set clamps to produce a normal mean arterial pressure of $93 \pm 1 \mathrm{~mm} \mathrm{Hg}$ and venous pressures of $9 \pm 2 \mathrm{~mm} \mathrm{Hg}$, each at its own sampling site, and we measured pressure with a disposable pressure transducer (DTX Plus DT-4812, Becton Dickinson, Franklin Lakes, New Jersey) connected via a fluid-filled circuit. We confirmed the accuracy of the transducer with a column of mercury. We employed a critical care monitor (S/5, DatexOhmeda, Madison, Wisconsin) to calculate and display the mean arterial and venous pressures.

We obtained 4 brands of arterial samplers with vented plungers (Portex Pro-Vent, safePICO [Radiometer America, Westlake, Ohio], Quik ABG [Vital Signs, Totowa, New Jersey], Pulset [WestMed, Tucson, Arizona]) and one brand of sampler without a plunger (ComfortSampler, OPTI Medical, Roswell, Georgia). We assigned each arterial sampler brand the numbers $1-5$ and the venous samplers the number 6 . We rolled a 6 -sided die to randomly select trials. We preset the samplers with plungers to $1 \mathrm{~mL}$ and used the 23-gauge/1-inch needle provided in the arterial sampler kits. For the sampler without a plunger, we used the 26-gauge/[7/8]-inch needle that was provided in the 
Table 1. Sampler Filling Times at a Mean Arterial Pressure of $93 \pm 1 \mathrm{~mm} \mathrm{Hg}$

\begin{tabular}{lccccc}
\hline \hline & $\begin{array}{c}\text { OPTI Medical } \\
\text { ComfortSampler }\end{array}$ & $\begin{array}{c}\text { WestMed } \\
\text { Pulset }\end{array}$ & $\begin{array}{c}\text { Vital Signs } \\
\text { Quik ABG }\end{array}$ & $\begin{array}{c}\text { Portex } \\
\text { Pro-Vent }\end{array}$ & $\begin{array}{c}\text { Radiometer } \\
\text { safePICO }\end{array}$ \\
\hline Arterial filling time (mean \pm SD), s & $5.1 \pm 0.5$ & $5.3 \pm 0.1$ & $5.6 \pm 0.3$ & $6.1 \pm 0.5$ & $6.2 \pm 0.2$ \\
\hline
\end{tabular}

arterial sampler kit. We inserted the sampler needle into the circuit at $45^{\circ}$ via a $4.0-\mathrm{mm}$ vascular access connector. For each sampler brand, we collected 10 samples at a pressure of $93 \pm 1 \mathrm{~mm} \mathrm{Hg}$ and 10 samples at a pressure of $9 \pm 2 \mathrm{~mm} \mathrm{Hg}$ for the sampler without a plunger. We used 2 stopwatches, and 2 investigators simultaneously timed the filling of the samplers. Timing began when the synthetic blood solution was first visualized in the needle hub and ended when the sampler stopped filling. Upon agreement of the 2 investigators, we accepted the filling times and recorded the average of the 2 times.

We used statistics software (SPSS 17.0, SPSS, Chicago, Illinois) for analysis of the data. To compare the filling times among the samplers with plungers, we computed the mean \pm SD and the Kruskal-Wallis one-way analysis of variance with $\alpha=.05$. To compare the filling times of the sampler without a plunger at venous and arterial pressures, we computed the mean $\pm \mathrm{SD}$ and a $t$ test for independent samples with $\alpha=.05$.

\section{Results}

The mean arterial sampler filling time for all 5 samplers combined was $5.7 \pm 0.6 \mathrm{~s}$ at a pressure of $93 \pm 1 \mathrm{~mm} \mathrm{Hg}$. The individual sampler filling rates are presented in Table 1 . The differences in filling rates among the 5 samplers were statistically significant $(P<.001)$. The mean filling times for the ComfortSampler were $97.7 \pm 30.7 \mathrm{~s}$ at a pressure of $9 \pm 2 \mathrm{~mm} \mathrm{Hg}$ and $5.1 \pm 0.5 \mathrm{~s}$ at a pressure of $93 \pm 1 \mathrm{~mm} \mathrm{Hg}$. This difference in filling times was statistically significant $(P<.001)$.

\section{Discussion}

Our study further supports previous studies conducted by Johnson et al, ${ }^{1}$ Bender et $a l,{ }^{2}$ and Cortes et al, ${ }^{3}$ who concluded that arterial sampler filling time can distinguish between arterial and venous punctures. Johnson et $\mathrm{al}^{1}$ used an extracorporeal laboratory model and tested various needle sizes, whereas Bender et $\mathrm{al}^{2}$ studied subjects with normal mean arterial pressures, and Cortes et $\mathrm{al}^{3}$ studied subjects with abnormal blood pressures. These studies used only a single brand of sampler; our study extends their work to all currently available samplers with and without plungers in the United States. Because Johnson et al, ${ }^{1}$ Bender et $\mathrm{al}^{2}{ }^{2}$ and Cortes et $\mathrm{al}^{3}$ already showed that there is a significant difference between arterial and venous fill- ing times with the Portex Pro-Vent, which is a sampler with a plunger, and because this sampler was included in our study, we chose to compare all samplers with a plunger at only mean arterial pressure. Although we found significant differences in sampler filling rates at a simulated mean arterial pressure between sampler brands, the difference of $\sim 1 \mathrm{~s}$ should not prevent distinguishing between arterial and venous punctures because the minimum difference between arterial and venous filling rates in all 3 previous studies was reported by Johnson et $\mathrm{al}^{1}$ as $51.4 \mathrm{~s}$. Currently, clinical practice guidelines suggest that blood color and pulsation may be used to identify arterial samples. ${ }^{5}$ Based on all 4 studies, arterial sampler filling rate may be a more objective indicator of obtaining an arterial sample and should be considered when guidelines are updated.

We used an extracorporeal circuit similar to that used by Johnson et $\mathrm{al}^{1}$, but our filling rate for the Portex Pro-Vent sampler with a 23-gauge needle at a normal mean arterial pressure was $6.1 \pm 0.5 \mathrm{~s}$, almost $10 \mathrm{~s}$ less compared with the rate obtained by Johnson et al. ${ }^{1}$ The reason for this difference may be that Johnson et $\mathrm{al}^{1}$ collected 2-mL volumes and used more viscous reconstituted human blood, whereas we collected $1 \mathrm{~mL}$ of a less viscous synthetic blood. Also contributing to the difference may be our lower blood flow. Johnson et $\mathrm{al}^{1}$ used a normal cardiac output of $5.0 \mathrm{~L} / \mathrm{min}$, whereas ours was $1.16 \mathrm{~L} / \mathrm{min}$. We reduced the flow to better mimic peripheral arterial flow. ${ }^{6}$

We used the 23-gauge needle for the samplers with a plunger and a 26-gauge needle for the Comfort-Sampler without a plunger. The ComfortSampler was originally available as the Roche Microsampler (Roche Diagnostics, Indianapolis, Indiana), and it was described and evaluated by d'Ortho et $\mathrm{al}^{4}$ in 2001 . Because the volume of the sampler was $240 \mu \mathrm{L}$, it took significantly less time to fill to capacity. The difference in filling times would have more likely been longer if blood volumes were equal. Because smaller needles may provide enhanced patient safety and comfort, sampler filling times when using vented samplers and needles smaller than 23 gauge should be studied further.

Vented arterial samplers were initially described in 1986 by Hutchison et al, ${ }^{7}$ who compared blood gas values between plastic vented Monovette samplers (Sarstedt, Newton, North Carolina) and glass syringes and found a significant increase in $\mathrm{P}_{\mathrm{O}_{2}}$ in the samplers. This difference was attributed to the blood being exposed to air during 
sampling and not removed before analysis. Hutchison et $\mathrm{al}^{7}$ iced all blood samples and did not report the pre-analytical time period; these variables have also been attributed to erroneously high $\mathrm{P}_{\mathrm{O}_{2}}{ }^{8}{ }^{8-10}$ Sampler filling time is also the time arterial blood is exposed to atmospheric air. If filling time is excessive, time exposed to air might have an effect on $\mathrm{P}_{\mathrm{O}_{2}}$ values. ${ }^{4}$ The impact of this time on current vented samplers has not been reported and deserves further study.

In addition, we tested one sampler without a plunger at both arterial and venous pressures to determine whether the plunger had an effect on filling time. We found that there was a statistically significant difference in the filling times between arterial and venous punctures with the sampler without a plunger. Therefore, distinguishing between arterial and venous punctures does not depend on the brand or design of the sampler used.

The limitations of this study include the use of an extracorporeal circuit in place of human subjects. Although we simulated mean arterial pressure in our circuit, it is not the same as natural pulsatile arterial blood flow because it is a constant pressure. Results in humans or with a pulsatile pump may differ. We were also limited by our use of normal saline-based synthetic blood solution instead of human blood. Further research could include the direct comparison of filling times for venous and arterial pressures among all sampler brands and the effect of smaller gauge needles. $\mathrm{P}_{\mathrm{O}_{2}}$ could also be measured to determine whether the different venting systems and filling times of the samplers used affect the results of blood gas analysis.

\section{Conclusions}

Although we found a statistically significant difference between arterial filling times among various samplers with plungers, the difference was $<1 \mathrm{~s}$ and was not deemed clinically important for distinguishing between arterial and venous punctures. Respiratory therapists and other clinicians performing arterial punctures can use any currently available vented arterial blood gas sampler and be able to identify a successful arterial puncture based on filling times or rate $(\mathrm{s} / \mathrm{mL})$.

\section{REFERENCES}

1. Johnson KL, Johnson AJ, Barnes KA, Douce FH. Effects of mean arterial pressure and needle size on arterial sampler filling time. Respir Care 2011;56(4):456-459.

2. Bender JJ, Allison JR, Goehring JJ, Patel MD, Niederst SM, Douce FH. Arterial sampler filling times during percutaneous puncture in human subjects. Respir Care 2012;57(11):1945-1948.

3. Cortes AL, Dalessandro CM, Glade TM, Shirdon SA, Uhlir JJ, Douce FH. The effects of abnormal blood pressure on arterial sample filling times. Respir Care 2013;58(11):1907-1910.

4. d'Ortho MP, Delclaux C, Zerah F, Herigault R, Adnot S, Harf A. Use of glass capillaries avoids the time changes in high blood $\mathrm{P}_{\mathrm{O}_{2}}$ observed with plastic syringes. Chest 2001;120(5):1651-1654.

5. American Association for Respiratory Care. AARC clinical practice guideline. Sampling for arterial blood gas analysis. Respir Care 1992; 37(8):913-917.

6. Olamaei N, Dupuis J, Ngo Q, Finnerty V, Vo Thang TT, Authier S, et al. Characterization and reproducibility of forearm arterial flow during hyperemia. Physiol Meas 2010;31(6):763-773.

7. Hutchison AS, Dryburgh FJ, Ralston SH. Sampling errors in $\mathrm{pH}$ and blood gas analysis-an evaluation of three new arterial blood samplers. Ann Clin Biochem 1986;23(Pt 3):329-333.

8. Knowles TP, Mullin RA, Hunter JA, Douce FH. Effects of syringe material, sample storage time, and temperature on blood gases and oxygen saturation in arterialized human blood samples. Respir Care 2006;51(7):732-736.

9. Smeenk FW, Janssen JD, Arends BJ, Harff GA, van den Bosch JA, Schönberger JP, Postmus PE. Effects of four different methods of sampling arterial blood and storage time on gas tensions and shunt calculation in the 100\% oxygen test. Eur Respir J 1997;10(4):910913.

10. Davis MD, Walsh BK, Sittig SE, Restrepo RD. AARC clinical practice guideline: blood gas analysis and hemoximetry: 2013. Respir Care 2013;58(10):1694-1703. 